\title{
Fluoroboric acid adsorbed on silica gel catalyzed synthesis of bisindolyl alkanes under mild and solvent-free conditions
}

\author{
B. P. Bandgar,* Abasaheb. V. Patil, and V. T. Kamble \\ Organic Chemistry Research Laboratory, School of Chemical Sciences, \\ Swami Ramanand Teerth Marathwada University, Nanded - 431606, Maharashtra, India. \\ E-mail: bandgarbp@yahoo.com
}

\begin{abstract}
Efficient electrophilic substitution of indoles with various carbonyl compounds and (1H-indol-3yl)(alkyl) methanol were carried out smoothly using catalytic amount of $\mathrm{HBF}_{4}-\mathrm{SiO}_{2}$ under solvent free conditions to afford the symmetrical and unsymmetrical bis (indolyl) alkanes in good to excellent yields respectively. The method is simple, cost effective and gives good yields in short reaction times with recyclable catalyst.
\end{abstract}

Keywords: Bisindolylmethanes, $\mathrm{HBF}_{4}-\mathrm{SiO}_{2}$, solvent-free reactions

\section{Introduction}

Indole and their derivatives have been identified as an important class of compounds in pharmaceuticals. ${ }^{1}$ During the past few years a large number of natural products containing bis(indolyl)methanes, and bis(indolyl)ethanes ${ }^{2}$ have been isolated from marine sources. Some of them have been found to have interesting biological activity. bis(indolyl)methane is the most cruciferous substances for promoting beneficial estrogen metabolism in women and men. ${ }^{3}$ Therefore, the synthesis of these moieties has become interesting targets to synthetic chemists. ${ }^{4}$

Common synthetic methods for the synthesis of bis(indolyl)alkanes include the electrophilc substitution of indoles with various aldehydes or ketones in presence of either protic or Lewis acids. $^{5-18}$ Although, the synthesis of symmetrical bis(indolyl)alkanes has been studied extensively throughout the last century, the synthesis of unsymmetrical bis(indolyl)alkanes is still highly sought-after in synthetic community.

Evolution of clean and environmentally benign chemical processes using less hazardous catalysts has become a primary goal in synthetic organic chemistry. In particular, running a reaction under heterogeneous condition is more promising since it involves the facile recovery and reuse of the catalyst. In this regard, we wish to report $\mathrm{HBF}_{4}-\mathrm{SiO}_{2}$ as reusable catalyst for the synthesis of bis(indolyl)methanes (Scheme 1). 


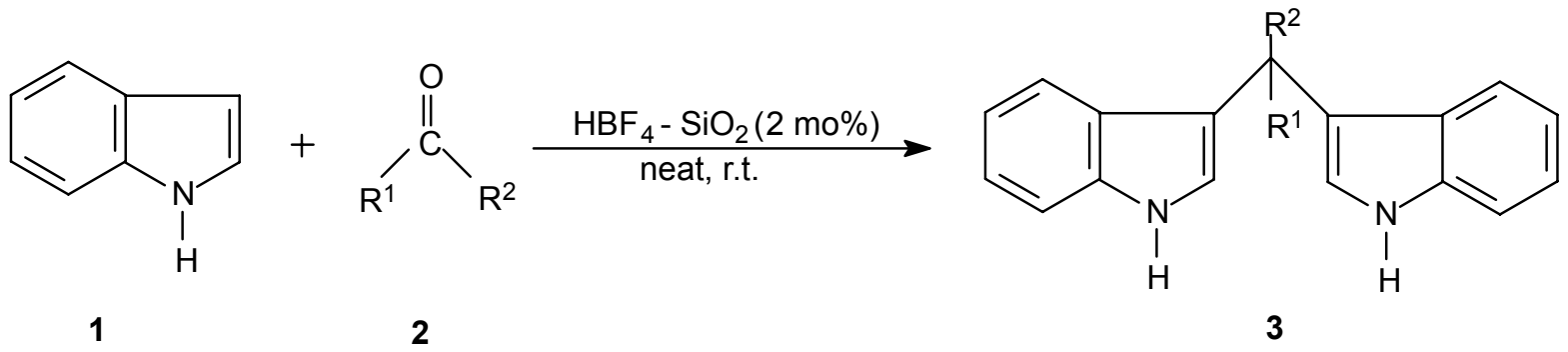

\section{Scheme 1}

\section{Results and Discussion}

Initially a systematic study was carried out for evaluation for $\mathrm{HBF}_{4}-\mathrm{SiO}_{2}$ as a catalyst for the reaction of indole with benzaldehyde under various conditions (Table 1). The reaction was slow in the absence of catalyst (table 1, entry 1) and inferior results were obtained in the presence of solvents (Table 1, entries 2-5). Next, we optimized the quantity of catalyst at room temperature under solvent-free conditions (Table 1, entries 6-11) and it was observed that the use of just 2 mol\% is sufficient to produce an excellent yield of the product (Table 1, entry 9) where as more than $2 \mathrm{~mol} \%$ of the catalyst did not improve the results (Table 1, entries 10-11).

Table 1. Reaction of indole with benzaldehyde under various conditions

\begin{tabular}{ccccc}
\hline Entry & Solvent & $\mathrm{HBF}_{4}-\mathrm{SiO}_{2},(\mathrm{~mol} \%)$ & $\mathrm{Time}, \mathrm{min} /[\mathrm{h}]$ & Yield, $(\%)$ \\
\hline 1. & neat & ------ & {$[20]$} & 10 \\
2. & $\mathrm{CH}_{2} \mathrm{Cl}_{2}$ & 2 & 50 & 75 \\
3. & $\mathrm{THF}$ & 2 & 55 & 65 \\
4. & $\mathrm{CH}_{3} \mathrm{CN}$ & 2 & 45 & 78 \\
5. & $\mathrm{CHCl}_{3}$ & 2 & 40 & 75 \\
6. & neat & 0.5 & 10 & 65 \\
7. & neat & 1.0 & 15 & 80 \\
8. & neat & 1.5 & 10 & 88 \\
9. & neat & 2 & 10 & 94 \\
10. & neat & 2.5 & 10 & 92 \\
11. & neat & 3.3 & 10 & 94 \\
\hline
\end{tabular}


Another advantage of the use of the catalyst was that it could be easily removed and recycled in subsequent reaction without significantly decreasing the activity of catalyst, by simple filtration and extraction. The catalyst could be recycled three times without substantial loss of activity (90\% $1^{\text {st }}$ run; $88 \% 2^{\text {nd }}$ run; $82 \% 3^{\text {rd }}$ run).

Further, we screened the reaction of indole with benzaldehyde in presence of $2 \mathrm{~mol} \% \mathrm{HBF}_{4}$ $\mathrm{SiO}_{2}$ as a catalyst at room temperature under solvent-free conditions. This furnished the corresponding bis(indolyl)methanes in 94\% yields. The electrophilic substitutions of indole with aldehydes as well as ketones proceeded smoothly at room temperature. In all cases aldehydes reacted more rapidly and gave higher yields than ketones. The scope and generality of the present method have been shown with respect to various carbonyl compounds (Table 2, entries a-k).

Then, we extended the reaction of indole with $1 H$-indole-3-yl- (phenyl)methanol and its analogs (Scheme 2) under similar conditions at room temperature, furnishing the unsymmetrical bis(indolyl)alkanes in good yields (Table 2, entries 1-O). Our findings reflect the wide applicability and usefulness of this method. In all cases, reaction proceeded efficiently to give the corresponding unsymmetrical bis(indolyl)alkanes in moderate yields. The reactions were clean and the products were obtained without the formation of any by-products.

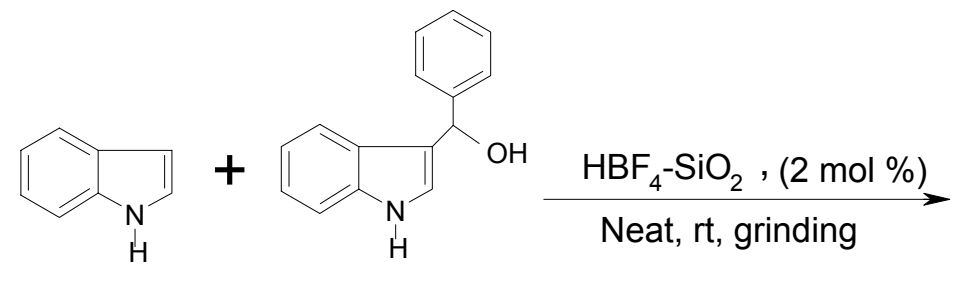

1

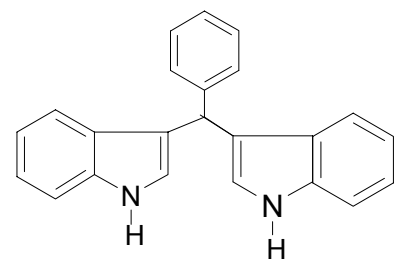

3

\section{Scheme 2}

\section{Conclusions}

In summary, we have developed a simple, novel and efficient synthetic protocol for the synthesis of symmetrical and unsymmetrical bis(indolyl)alkanes using catalytic amount of $\mathrm{HBF}_{4}-\mathrm{SiO}_{2}$ under solvent free conditions at room temperature by grinding. The short reaction time coupled with the simplicity of the reaction procedure, inexpensive and reusable catalyst make this method one of the most efficient methods for the synthesis of this class of compounds. 
Table 2. $\mathrm{HBF}_{4}-\mathrm{SiO}_{2}$ catalyzed synthesis of $\mathrm{Bis}(\mathrm{indolyl})$ methanes

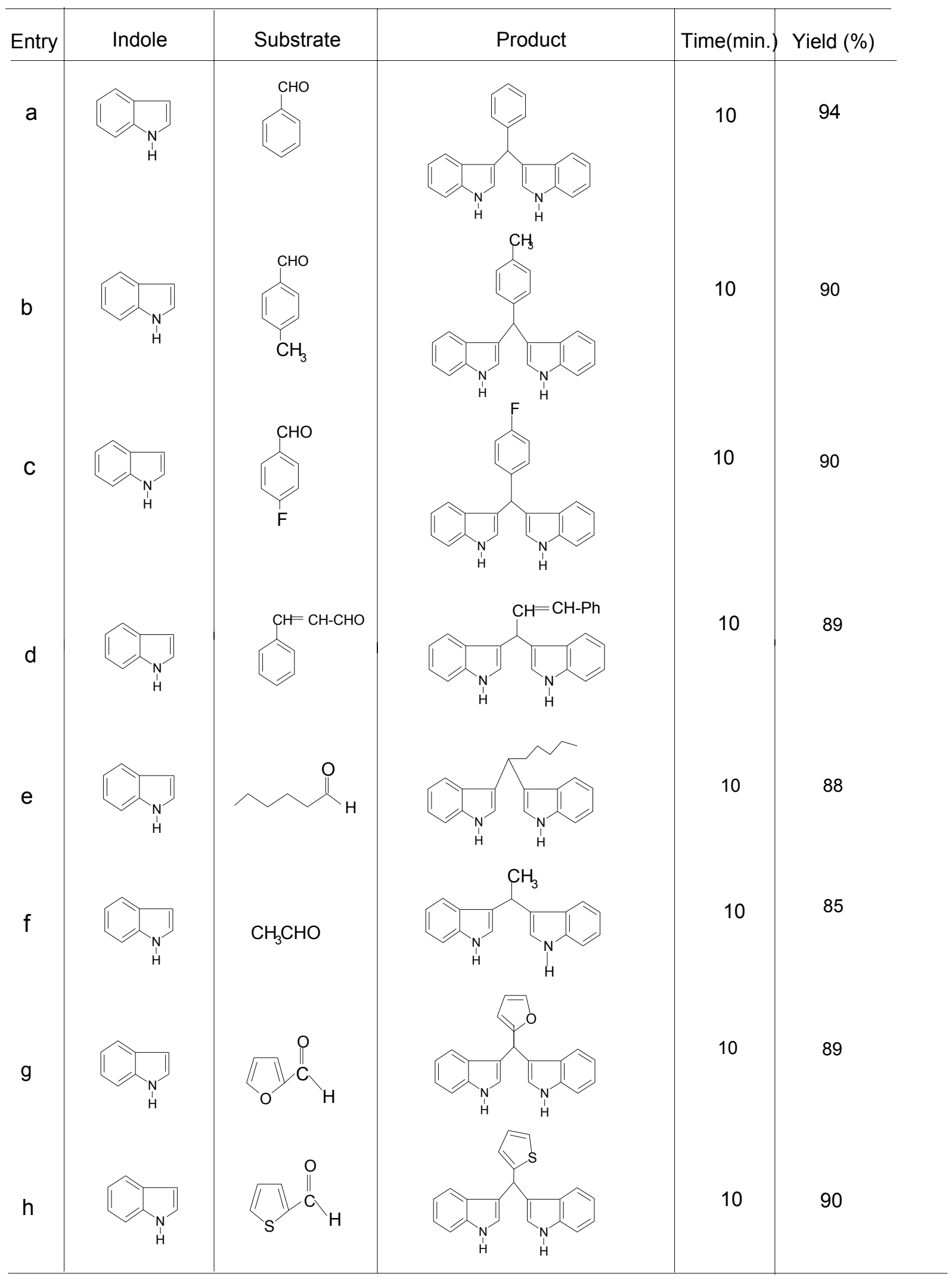




En try

\section{Experimental Section}

Preparation of catalyst. The catalyst was prepared by following literature procedure. ${ }^{19} \mathrm{HBF}_{4}$ (1.65 g, as a 40\% aqueous solution) was added to the suspension of silica gel (13.35 g, 230-400 mesh) in diethyl ether $(40 \mathrm{~mL})$. The mixture was concentrated and the residue dried under vaccum at $100{ }^{0} \mathrm{C}$ for $72 \mathrm{~h}$ to afford $\mathrm{HBF}_{4}-\mathrm{SiO}_{2}\left(0.5 \mathrm{mmolg}^{-1}\right)$ as a free flowing powder.

Preparative procedure. In a mortar, to a mixture of indole $(2 \mathrm{mmol})$ and benzaldehyde (1.2 $\mathrm{mmol}$ ), $\mathrm{HBF}_{4}-\mathrm{SiO}_{2}(40 \mathrm{mg}, 2 \mathrm{~mol} \%$ ) was added with constant and vigorous stirring for the required time (Table 1). After completion of the reaction (TLC), the reaction mixture was diluted with diethyl ether $(20 \mathrm{ml})$ and the catalyst was separated by filtration. The filtrate was dried over 
anhydrous $\mathrm{Na}_{2} \mathrm{SO}_{4}$ and then evaporated on vacuum to give crude product, which on further purification by column chromatography yielded the corresponding product in almost quantitative yield. The recovered catalyst was further utilized for three runs without substantial loss of activity, yielding $90,88,82 \%$ yield of the product.

\section{Physical and spectral data of the products}

1H, 1'H-3,3'-Phenyl methanediyl- bis-indole (3a). Colorless needles; mp 151-152 ${ }^{\circ} \mathrm{C}$; IR (KBr): 3387, 3047, 2957, 2927, 1482, 1456, 1340, 1095, $736 \mathrm{~cm}^{-1} ;{ }^{1} \mathrm{H}$ NMR (200 MHz, $\left.\mathrm{CDCl}_{3}\right)$ : $\delta 5.82(\mathrm{~s}, 1 \mathrm{H}), 6.46(\mathrm{~s}, 2 \mathrm{H}), 6.95(\mathrm{t}, 2 \mathrm{H}, J=6.0 \mathrm{~Hz}), 7.10-7.36(\mathrm{~m}, 11 \mathrm{H}), 7.78(\mathrm{brs}, 2 \mathrm{H}) ;{ }^{13} \mathrm{C}$ $\operatorname{NMR}\left(50 \mathrm{MHz}, \mathrm{CDCl}_{3}\right): \delta=29.62,40.02,111.06,118.99,119.71,121.67,123.63,126,126.88$, 128.11, 128.59, 136.50, 144;EIMS $m / z 322\left(\mathrm{M}^{+}\right)$; Anal. Calcd for $\mathrm{C}_{23} \mathrm{H}_{18} \mathrm{~N}_{2}$ : C, 85.68; H, 5.62; N, 8.68. Found: C, 85.75; H, 5.56; N, 8.56;

1H, 1'H-3,3'-(4-Methyl phenyl methanediyl)- bis-indole (3b). Pink colored solid; mp 94$96^{\circ} \mathrm{C}$; IR (KBr): 3410, 3040, 2930, 1600, 1510, 1215, 1050, $775 \mathrm{~cm}^{-1}$; ${ }^{1} \mathrm{H}$ NMR (200 MHz, $\left.\mathrm{CDCl}_{3}\right): \delta 2.32(\mathrm{~s}, 3 \mathrm{H}), 5.86(\mathrm{~s}, 1 \mathrm{H}), 6.68(\mathrm{~s}, 2 \mathrm{H}), 7.02(\mathrm{t}, 2 \mathrm{H}, J=7.2 \mathrm{~Hz}), 7.1(\mathrm{~d}, 2 \mathrm{H}, \mathrm{J}=7.2$ $\mathrm{Hz}$ ), 7.23-7.27 (m, 6H), 7.40 (d, 2H, J = 7.2 Hz), 7.93 (brs, 2H); EIMS m/z $336\left(\mathrm{M}^{+}\right)$; Anal. Calcd for $\mathrm{C}_{24} \mathrm{H}_{20} \mathrm{~N}_{2}$ : C, 85.68; H, 5.99; N, 8.32. Found: C, 85.37; H, 5.95; N, 8.14;

1H, 1'H-3,3'-(4-Fluorophenyl methanediyl) bis-indole (3c). Pink solid; mp 80-82 ${ }^{\circ} \mathrm{C}$; IR (KBr): 3410, 3054, 1487, 1455, $1089 \mathrm{~cm}^{-1} ;{ }^{1} \mathrm{H}$ NMR (200 MHz, $\left.\mathrm{CDCl}_{3}\right): \delta 5.86(\mathrm{~s}, 1 \mathrm{H}), 6.66(\mathrm{~s}$, 2H), $7.02(\mathrm{t}, 2 \mathrm{H}, J=8.3 \mathrm{~Hz}), 7.18(\mathrm{t}, 2 \mathrm{H}, \mathrm{J}=7.9 \mathrm{~Hz}), 7.26-7.38$ (m, 8H), 7.98 (brs, 2H); EIMS $m / z 340\left(\mathrm{M}^{+}\right)$; Anal. Calcd for $\mathrm{C}_{23} \mathrm{H}_{17} \mathrm{~N}_{2} \mathrm{~F}: \mathrm{C}, 81.15 ; \mathrm{H}, 5.03 ; \mathrm{N}, 8.22 ; \mathrm{F}, 5.58$. Found : C, 81.30; H, 5.18; N, 8.40; F, 5.69.

1H, 1'H-3,3'-Cinnamylmethanediyl bis-indole (3d). Semi solid; IR (KBr): 3450, 3100, 2960, 1590, 1470, 1030, 970, $760 \mathrm{~cm}^{-1} ;{ }^{1} \mathrm{H}$ NMR $\left(200 \mathrm{MHz}, \mathrm{CDCl}_{3}\right): \delta 5.90(\mathrm{~s}, 1 \mathrm{H}), 6.45(\mathrm{~d}, 1 \mathrm{H}, J=$ $16.6 \mathrm{~Hz}), 6.65(\mathrm{~d}, 2 \mathrm{H}, J=2.3 \mathrm{~Hz}), 7.05(\mathrm{t}, 2 \mathrm{H}, J=8.1 \mathrm{~Hz}), 7.15(\mathrm{t}, 2 \mathrm{H}, J=8.1 \mathrm{~Hz}), 7.25(\mathrm{~m}$, 4H), 7.35 (m, 3H), 7.55 (m, 2H), 7.75 (d, 1H, J=16.6 Hz), 7.85 (brs, 2H); EIMS m/z 348 ;(M ); Anal. Calcd for $\mathrm{C}_{25} \mathrm{H}_{20} \mathrm{~N}_{2}$ : C, 86.17; H, 5.78; N, 8.03. Found : C, 86.28; H, 5.60; N, 8.13;

$\mathbf{1 H}, \mathbf{1}^{\prime} \boldsymbol{H}-3,3$ '-Hexanylmethanediyl bis-indole (3e). Solid; mp $68-70^{\circ} \mathrm{C}$; IR (KBr): 3500, 3100, 3030, 2950, 1610, 1580, 1250, 1060, $770 \mathrm{~cm}^{-1} ;{ }^{1} \mathrm{H}$ NMR $\left(200 \mathrm{MHz}, \mathrm{CDCl}_{3}\right): \delta 0.8(\mathrm{t}, 3 \mathrm{H}, J=6.8$ $\mathrm{Hz}), 1.25(\mathrm{~m}, 6 \mathrm{H}), 2.25(\mathrm{~m}, 2 \mathrm{H}), 4.60(\mathrm{t}, 1 \mathrm{H}, J=6.8 \mathrm{~Hz}), 6.85(\mathrm{~d}, 2 \mathrm{H}, J=2.3 \mathrm{~Hz}), 7.05(\mathrm{t}, 2 \mathrm{H}, J$ $=8.0 \mathrm{~Hz}), 7.15(\mathrm{t}, 2 \mathrm{H}, J=8 \mathrm{~Hz}), 7.35(\mathrm{~d}, 2 \mathrm{H}, J=8 \mathrm{~Hz}), 7.50(\mathrm{~d}, 2 \mathrm{H}, J=8 \mathrm{~Hz}), 7.85$ (brs, 2H); EIMS $m / z 316\left(\mathrm{M}^{+}\right)$; Anal. Calcd for $\mathrm{C}_{22} \mathrm{H}_{24} \mathrm{~N}_{2}$ : C, 83.50; $\mathrm{H}, 7.64 ; \mathrm{N}, 8.85$. Found : C, 83.65; $\mathrm{H}$, $7.67 ; \mathrm{N}, 8.83$;

1H, 1'H-3,3'-((1-Methyl)methanediyl) bis-indole (3f). Red powder; mp $92^{\circ} \mathrm{C}$; IR (KBr): 3399 , 3389, $2958 \mathrm{~cm}^{-1}$; ${ }^{1} \mathrm{H}$ NMR $\left(200 \mathrm{MHz}, \mathrm{CDCl}_{3}\right): \delta 1.91(\mathrm{~d}, 3 \mathrm{H}, J=6.8 \mathrm{~Hz}), 4.5(\mathrm{~m}, 1 \mathrm{H}), 6.85(\mathrm{t}$, $2 \mathrm{H}, J=6.8 \mathrm{~Hz}), 7.03(\mathrm{~m}, 2 \mathrm{H}), 7.07(\mathrm{t}, 2 \mathrm{H}, J=8 \mathrm{~Hz}), 7.25(\mathrm{~d}, 2 \mathrm{H}, J=8 \mathrm{~Hz}), 7.40$ (d, 2H, $J=8$ $\mathrm{Hz}$ ), 7.84 (brs, 2H); ; EIMS $m / z 260\left(\mathrm{M}^{+}\right)$; Anal. Calcd for $\mathrm{C}_{18} \mathrm{H}_{16} \mathrm{~N}_{2}$ : C, 83.04; H, 6.19; N, 10.75. Found : C, 83.25; H, 6.26; N, 10.56;

1H, 1'H-3,3'-(2- Furanyl methanediyl) bis-indole (3g). Brown colored solid; mp 324-326 ${ }^{\circ} \mathrm{C}$; IR (KBr): 3410, 1715, 1450, $1260 \mathrm{~cm}^{-1} ;{ }^{1} \mathrm{H}$ NMR (200 MHz, $\left.\mathrm{CDCl}_{3}\right): \delta 5.95$ (s, 1H), 6.90 (s, 
2H), 7.03-7.50 (m, 11H), 8.0 (brs, 2H); EIMS m/z $264\left(\mathrm{M}^{+}\right)$; Anal. Calcd for $\mathrm{C}_{17} \mathrm{H}_{16} \mathrm{~N}_{2} \mathrm{O}: \mathrm{C}$, 77.24; H, 6.10; N, 10.59. Found: C, 77.35; H, 6.26; N, 10.56;

1H, 1'H-3,3'-Thienyl methanediyl bis-indole (3h). Brown solid; mp 151- $152^{\circ} \mathrm{C}$; IR (KBr): 3410, 1715, 1450, $1260 \mathrm{~cm}^{-1} ;{ }^{1} \mathrm{H}$ NMR $\left(200 \mathrm{MHz}, \mathrm{CDCl}_{3}\right): \delta 6.18(\mathrm{~s}, 1 \mathrm{H}), 6.87(\mathrm{~s}, 2 \mathrm{H}), 6.92-$ 7.48 (m, 11H), 7.98 (brs, 2H); EIMS m/z $280\left(\mathrm{M}^{+}\right)$; Anal. Calcd for $\mathrm{C}_{17} \mathrm{H}_{16} \mathrm{~N}_{2} \mathrm{~S}: \mathrm{C}, 72.82 ; \mathrm{H}$, 5.75; N, 9.99. Found: C, 72.62; H, 5.70; N, 9.84;

1H, 1'H-3,3'-Benzo[1,3]dioxol-5yl methanediyl bis-indole (3i). Yellow solid; mp 89-91 ${ }^{\circ} \mathrm{C}$; IR (KBr): 3410, 1715, 1450, $1260 \mathrm{~cm}^{-1} ;{ }^{1} \mathrm{H}$ NMR (200 MHz, $\left.\mathrm{CDCl}_{3}\right): \delta 5.82(\mathrm{~s}, 1 \mathrm{H}), 5.92(\mathrm{~s}, 2 \mathrm{H})$, $6.70(\mathrm{~s}, 2 \mathrm{H}), 6.74(\mathrm{~d}, 1 \mathrm{H}, J=8.2 \mathrm{~Hz}), 6.84(\mathrm{~d}, 2 \mathrm{H}, J=8.2 \mathrm{~Hz}), 7.02(\mathrm{t}, 2 \mathrm{H}, J=7.3 \mathrm{~Hz}), 7.18(\mathrm{t}$, $2 \mathrm{H}, J=7.3 \mathrm{~Hz}), 7.36-7.42(\mathrm{~m}, 4 \mathrm{H}), 7.95$ (brs, 2H); EIMS $\mathrm{m} / z 366\left(\mathrm{M}^{+}\right)$; Anal. Calcd for $\mathrm{C}_{24} \mathrm{H}_{18} \mathrm{~N}_{2} \mathrm{O}_{2}: \mathrm{C}, 78.67 ; \mathrm{H}, 4.95 ; \mathrm{N}, 7.64$. Found : C, 78.75; H, 4.82; N, 7.52;

1H, 1'H-3,3'-((1,1-Dimethyl)methanediyl) bis-indole (3j). Colorless needles; mp 64-66 ${ }^{\circ} \mathrm{C}$; IR (KBr): 3440, 2964, 2859, 1659, 1384, $734 \mathrm{~cm}^{-1} ;{ }^{1} \mathrm{H}$ NMR (200 MHz, $\left.\mathrm{CDCl}_{3}\right): \delta 1.91$ (s 6H), 6.87 $(\mathrm{t}, 2 \mathrm{H}, J=6.8 \mathrm{~Hz}), 7.02(\mathrm{~m}, 2 \mathrm{H}), 7.07(\mathrm{t}, 2 \mathrm{H}, J=8 \mathrm{~Hz}), 7.28(\mathrm{~d}, 2 \mathrm{H}, J=8 \mathrm{~Hz}), 7.40(\mathrm{~d}, 2 \mathrm{H}, J=8$ $\mathrm{Hz}$ ), 7.85 (brs, 2H); EIMS m/z $274\left(\mathrm{M}^{+}\right)$; Anal. Calcd for $\mathrm{C}_{19} \mathrm{H}_{18} \mathrm{~N}_{2}: \mathrm{C}, 83.17 ; \mathrm{H}, 6.61$; N, 10.62 . Found: C, 83.25; H, 6.56; N, 10.56;

$\mathbf{1 H}, \mathbf{1}^{\prime} \boldsymbol{H}-3,3$ '-Cyclohexanediyl-bis-indole (3k). Colorless needles; mp $118-120^{\circ} \mathrm{C}$; IR (KBr): 3450, 3030, 2929, 1658, 1550, 1461, $740 \mathrm{~cm}^{-1} ;{ }^{1} \mathrm{H}$ NMR (200 MHz, $\left.\mathrm{CDCl}_{3}\right): \delta 1.61(\mathrm{~m}, 6 \mathrm{H})$, $2.54(\mathrm{~m}, 4 \mathrm{H}), 3.25(\mathrm{~m}, 1 \mathrm{H}), 4.60(\mathrm{~s}, 1 \mathrm{H}), 6.89(\mathrm{t}, 2 \mathrm{H}, J=7.2 \mathrm{~Hz}), 7.07(\mathrm{~m}, 4 \mathrm{H}), 7.29(\mathrm{~d}, 2 \mathrm{H}, J=$ $8.1 \mathrm{~Hz}), 7.55$ (d, $2 \mathrm{H}, J=8.1 \mathrm{~Hz}), 7.92$ (brs, 2H); EIMS $m / z 326\left(\mathrm{M}^{+}\right)$; Anal. Calcd for $\mathrm{C}_{23} \mathrm{H}_{24} \mathrm{~N}_{2}$ : C, 84.62; H, 6.79; N, 8.58. Found: C, 84.43; H, 6.68; N, 8.44;

1H, 1'H-3,3'-Phenyl methanediyl-bis-indole (3I). Colorless needles; mp 151-152 ${ }^{\circ} \mathrm{C}$; IR (KBr): 3387, 3047, 2957, 2927, 1482, 1456, 1340, 1095, $736 \mathrm{~cm}^{-1} ;{ }^{1} \mathrm{H}$ NMR (200 MHz, $\left.\mathrm{CDCl}_{3}\right): \delta 5.82$ $(\mathrm{s}, 1 \mathrm{H}), 6.46(\mathrm{~s}, 2 \mathrm{H}), 6.95(\mathrm{t}, 2 \mathrm{H}, J=6.0 \mathrm{~Hz}), 7.10-7.36(\mathrm{~m}, 11 \mathrm{H}), 7.78(\mathrm{brs}, 2 \mathrm{H}) ;{ }^{13} \mathrm{C}$ NMR $(50$ $\left.\mathrm{MHz}, \mathrm{CDCl}_{3}\right): \delta=29.62,40.02,111.06,118.99,119.71,121.67,123.63,126,126.88,128.11$, 128.59, 136.50, 144;EIMS m/z $322\left(\mathrm{M}^{+}\right)$; Anal. Calcd for $\mathrm{C}_{23} \mathrm{H}_{18} \mathrm{~N}_{2}$ : C, 85.68; H, 5.62; N, 8.68. Found: C, 85.75; H, 5.56; N, 8.56;

3-((5-Methyl-1H-indol-3yl) (phenyl) methyl)-1H-indole (3m). Brown solid; mp 87-89 ${ }^{\circ} \mathrm{C}$; IR (KBr): 3409, 2960, $2842 \mathrm{~cm}^{-1} ;{ }^{1} \mathrm{H}$ NMR (200 MHz, $\left.\mathrm{CDCl}_{3}\right): \delta 2.49$ (s 3H), $5.89(\mathrm{~s}, 1 \mathrm{H}), 6.66$ (s, 2H), 6.92-7.03 (m, 3H), 7.16-7.30 (m, 5H), 7.35-7.41 (m, 4H), 7.83 (s, 1H), 7.90 (s, 1H); EIMS $m / z 336\left(\mathrm{M}^{+}\right)$; Anal. Calcd for $\mathrm{C}_{24} \mathrm{H}_{20} \mathrm{~N}_{2}$ : C, 85.68; H, 5.99; N, 8.32. Found: C, 85.75; H, 5.79; $\mathrm{N}, 8.46$;

3-((5-Bromo-1H-indol-3yl) (phenyl) methyl)-1H-indole (3n). Brown powder; $\mathrm{mp} 145-147^{\circ} \mathrm{C}$; IR (KBr): 3406, 3385, 2925, $2848 \mathrm{~cm}^{-1} ;{ }^{1} \mathrm{H}$ NMR (200 MHz, $\left.\mathrm{CDCl}_{3}\right): \delta 5.87(\mathrm{~s} 1 \mathrm{H}), 6.61(\mathrm{~s}$, 2H), $6.66(\mathrm{~s}, 1 \mathrm{H}), 7.0(\mathrm{~s}, 1 \mathrm{H}), 7.01(\mathrm{~s}, 1 \mathrm{H}), 7.06-7.35(\mathrm{~m}, 5 \mathrm{H}), 7.37-7.40(\mathrm{~m}, 4 \mathrm{H}), 7.82(\mathrm{~s}, 1 \mathrm{H})$, $7.90(\mathrm{~s}, 1 \mathrm{H})$; EIMS $m / z 401\left(\mathrm{M}^{+}\right)$; Anal. Calcd for $\mathrm{C}_{23} \mathrm{H}_{17} \mathrm{~N}_{2} \mathrm{Br}: \mathrm{C}, 68.83 ; \mathrm{H}, 4.27$; N, 6.98 . Found: C, 68.75; H, 4.35; N, 6.85;

3-((5-Nitro-1H-indol-3yl) (Phenyl) methyl)-1-tosyl-1H-indole (3o). Pink powder; mp 240$242^{\circ} \mathrm{C}$; IR (KBr): 3430, 2919, $2858 \mathrm{~cm}^{-1} ;{ }^{1} \mathrm{H}$ NMR (200 MHz, $\mathrm{CDCl}_{3}$ ): $\delta 2.32$ (s 3H), 5.71 (s, $1 \mathrm{H}), 6.52(\mathrm{~s}, 2 \mathrm{H}), 7.04(\mathrm{~s}, 2 \mathrm{H}), 7.11(\mathrm{~d}, 2 \mathrm{H}, J=6.8 \mathrm{~Hz}), 7.20(\mathrm{~d}, 2 \mathrm{H}, J=8.4 \mathrm{~Hz}), 7.24-7.29$ (m, 
8H), $7.64(\mathrm{~d}, 2 \mathrm{H}, J=8.0 \mathrm{~Hz}), 7.98(\mathrm{~d}, 1 \mathrm{H}, J=8.4 \mathrm{~Hz})$; EIMS $m / z$ 367( $\left.\mathrm{M}^{+}\right)$; Anal. Calcd for $\mathrm{C}_{23} \mathrm{H}_{23} \mathrm{~N}_{3} \mathrm{O}_{2}$ : C, 75.19; H, 4.66; N, 11.43. Found: C, 75.25; H, 4.72; N, 11.52.

\section{References}

1. (a) Carey, F. A.; Sundberg, R. J. Advanced Organic Chemistry; Part B, Plenum Press: New York. (b) Sundberg, R. J. The chemistry of Indoles; Academic: New York, 1970.

2. (a) Morris, S. A.; Anderson, R. J. Brominated bis(indole)alkaloids from the marine sponge Hexadella sp. Tetrahedron 1990, 46, 715. (b) Bifulco, G.; Bruno, I.; Riccio, R.; Lavayre, J.; Bourdy, G. Further brominated bis- and tris-indole alkaloids from the deep-water new Caldonain marine sponge Orina sp. J. Nat. Prod. 1995, 58, 1254.

3. Zeligs, M. A. J. Med. Food 1998, 1, 67.

4. Chakraborty, M.; Ramkrishna, B.; Yosihiro, H. Heterocycles 2001, 55, 2431.

5. Chaterjee, A.; Manna, S.; banerji, J. J. Chem. Soc., Perkin Trans. 1 1980, 553.

6. D'aurio, M. Tetrahedron 1991, 47, 9225.

7. Yadav, J. S.; Reddy, B. V. S.; Murthy, Ch. V. S. R.; Mahesh Kumar, G.; Madan, Ch. Synthesis 2001, 783.

8. Chakraborty, M.; Ghosh, N.; Basak, R.; Yoshihiro, H. Tetrahedron Lett. 2002, 43, 4075.

9. Koshima, H.; Matsusaka, W. J. Heterocycl. Chem. 2002, 39, 1089.

10. Bandgar, B. P.; Shaikh, K. A. Tetrahedron Lett. 2003, 44, 1959.

11. Jun-ji, S.; Zhou, M. F.; Gu, D. G.; Wang, S. Y.; Loh, T. P. Synlett 2003, 2077.

12. Mi. X.; Lou, S.; He, J.; Cheng, J. P. Tetrahedron Lett. 2004, 45, 4567.

13. Mi, X.; Lou, S.; He, J.; Cheng, J. P. Eur. J. Org. Chem. 2004, 1584.

14. Feng, X. L.; Guan, C. J.; Zhao, C. X. Synth. Commun. 2004, 34, 487.

15. Ji, S. J.; Wang, S.Y.; Zhang, Y.; Loh, T. P. Tetrahedron 2004, 60, 2051.

16. Wang, L.; Han, J.; Tian, H.; Sheng, J.; Fan, Z.; Tang, X. Synlett 2005, 337.

17. Singh, P. R.; Singh, D. U.; Samant, S. D. Synth. Commun. 2003, 33, 2133.

18. Zeng, X. F.; Ji, S. J.; Wang, S. Y. Tetrahedron 2005, 61, 10235.

19. Chakraborti, A. K.; Gulhane, R. Tetrahedron Lett. 2003, 44, 3521. 